CRYSTALLOGRAPHIC COMMUNICATIONS

ISSN 2056-9890

Received 25 February 2016

Accepted 3 March 2016

Edited by D.-J. Xu, Zhejiang University (Yuquan Campus), China

Keywords: crystal structure; hydrogen bond; $(E, E)-2^{\prime}, 4^{\prime}$-dihydroxyacetophenone azine.

CCDC reference: 1457201

Supporting information: this article has supporting information at journals.iucr.org/e

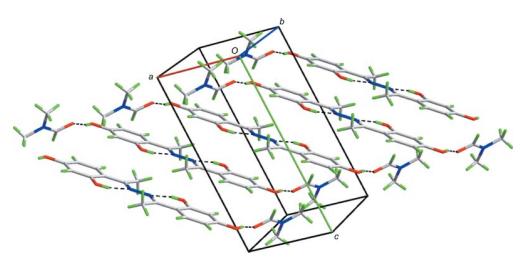

OPEN $\odot$ ACCESS

\section{Crystal structure of $(E, E)-2^{\prime}, 4^{\prime}$-dihydroxyaceto- phenone azine dimethylformamide disolvate}

\author{
Wen-Juan Li and Hong-Fei Han*
}

Department of Chemistry, Taiyuan Normal University, Taiyuan 030031, People's Republic of China. *Correspondence e-mail: hfhan001@163.com

In the title compound \{systematic name: 4,4'-[1,1'-(hydrazinediylidene)bis(ethan-1-yl-1-ylidene)]bis(benzene-1,3-diol)\}, $\mathrm{C}_{16} \mathrm{H}_{16} \mathrm{~N}_{2} \mathrm{O}_{4} \cdot 2 \mathrm{C}_{3} \mathrm{H}_{7} \mathrm{NO}$, the (E,E)$2^{\prime}, 4^{\prime}$-dihydroxyacetophenone azine molecule is centrosymmetric, the mid-point of the $\mathrm{N}-\mathrm{N}$ bond being located on an inversion centre. All the non-H atoms of the azine molecule are approximately coplanar, the maximum deviation being 0.017 (2) $\AA$. An intramolecular $\mathrm{O}-\mathrm{H} \cdots \mathrm{N}$ hydrogen bond occurs between the azine $\mathrm{N}$ atom and the hydroxy group. In the crystal, azine and dimethylformamide solvent molecules are linked by $\mathrm{O}-\mathrm{H} \cdots \mathrm{O}$ hydrogen bonds.

\section{Chemical context}

Hydrazones are important compounds due to their possible applications in material and coordination chemistry. Fluorescence properties of hydrazones have been reported (Qin et al., 2009). Many organometallic compounds containing acylhydrazone ligands have also been synthesized for their potential magneto-chemical properties (Guo et al., 2010). In particular, they have received increasing interest for their biological activity as antioxidants (Kitaev et al., 1970), and their antimicrobial (Ramamohan et al., 1995) and antiviral properties (El-Tabl et al., 2008; Rollas \& Küçükgüzel, 2007).

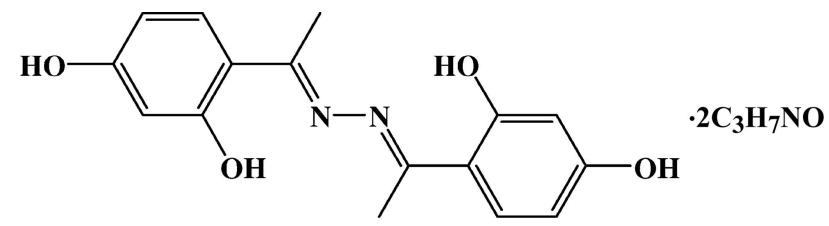

Although 2',4'-dihydroxyacetophenone azine has been prepared and studied as a fluorescent probe, its structure has not been reported. As a part of our studies on synthesis and structural peculiarities of Schiff base ligands derived from $2^{\prime}, 4^{\prime}$-dihydroxyacetophenone and hydrazine, we determined the structure of the title compound, $(E, E)-2^{\prime}, 4^{\prime}$-dihydroxyacetophenone azine dimethylformamide disolvate, (I).

\section{Structural commentary}

The molecular structure of the title compound is depicted in Fig. 1. The asymmetric unit contains one half-molecule of $(E, E)-2^{\prime}, 4^{\prime}$-dihydroxyacetophenone azine and one dimethylformamide (DMF) molecule. The complete azine molecule is centrosymmetric and exists in an $E, E$ configuration with respect to the two $\mathrm{C}=\mathrm{N}$ bonds. The $\mathrm{N} 1-\mathrm{C} 2$ bond length of 1.301 (3) $\AA$ shows double-bond character. The $\mathrm{C}-\mathrm{O}$ bond lengths $[1.349(3)$ and $1.358(3) \AA]$ are comparable with 


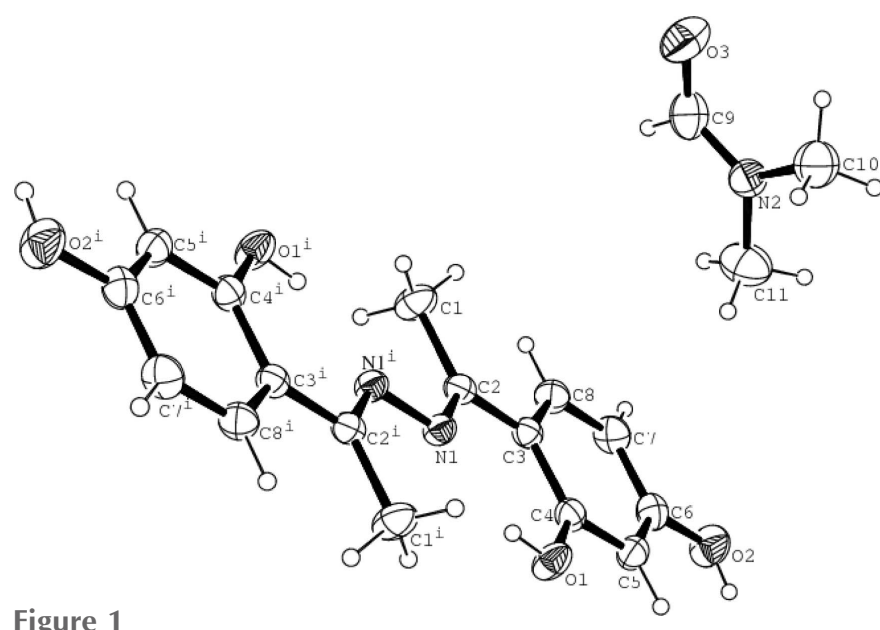

Figure

The molecular structure of the title compound, showing the atomnumbering scheme. Displacement ellipsoids are drawn at the $30 \%$ probability level. Only one DMF solvent molecule is shown. [Symmetry code: (i) $-x+1,-y+1,-z+1$.]

similar bonds in related structures (Chantrapromma et al., 2011; Tai et al., 2008). All the non-H atoms of the azine molecule are approximately coplanar. The nine atoms (i.e. N1, C1 and $\mathrm{C} 2$, and the six $\mathrm{C}$ atoms in the benzene ring) are essentially planar, with a mean deviation of $0.0024 \AA$. Each hydroxy group is nearly coplanar with its attached benzene ring; the r.m.s. deviation is $0.0045 \AA$ for the seven non-H atoms. Intramolecular $\mathrm{O}-\mathrm{H} \cdots \mathrm{N}$ hydrogen bonds exist in the azine molecule (Table 1).

\section{Supramolecular features}

In the crystal of (I), intermolecular $\mathrm{O}-\mathrm{H} \cdots \mathrm{O}$ hydrogen bonds exist between azine molecules and DMF molecules (Table 1 and Fig. 2).
Table 1

Hydrogen-bond geometry $\left(\AA{ }^{\circ}\right)$.

\begin{tabular}{lllll}
\hline$D-\mathrm{H} \cdots A$ & $D-\mathrm{H}$ & $\mathrm{H} \cdots A$ & $D \cdots A$ & $D-\mathrm{H} \cdots A$ \\
\hline $\mathrm{O} 1-\mathrm{H} 1 \cdots \mathrm{N} 1$ & 0.82 & 1.82 & $2.543(2)$ & 147 \\
$\mathrm{O}^{\mathrm{i}}-\mathrm{H} 2 \cdots \mathrm{O}^{\mathrm{i}}$ & 0.82 & 1.84 & $2.649(3)$ & 171 \\
\hline
\end{tabular}

Symmetry code: (i) $x-1, y+1, z$.

\section{Database survey}

A search of Cambridge Structural Database (Groom \& Allen, 2014) for acetophenone azine gave 105 hits (excluding organometallics). There are four reported crystal structures of acetophenone azine containing hydroxy groups at the 2position of benzene rings: (E,E)-2,2'-(1,1'-azinodiethylidyne)diphenol (Tai et al., 2008), (E,E)-4,4'-dichloro-2,2'-(1,1'azinodiethylidyne)diphenol (Chang et al., 2007), (E,E)-3,3'diethoxy-2,2'-(1,1'-azinodiethylidyne)diphenol (Fayos et al., $1980)$ and $(E, E)-4,4^{\prime}$-dimethoxy-2,2'-(1,1'-azinodiethylidyne)diphenol (Zhang et al., 2008).

\section{Synthesis and crystallization}

A mixture of $2^{\prime}, 4^{\prime}$-dihydroxyacetophenone (3.06 g, $\left.20 \mathrm{mmol}\right)$, hydrazine sulfate $(1.28 \mathrm{~g}, 10 \mathrm{mmol})$ and triethylamine $(3.03 \mathrm{~g}$, $30 \mathrm{mmol})$ in ethanol $(40 \mathrm{ml})$ was heated under reflux for $24 \mathrm{~h}$. After cooling, the precipitate was filtrated and washed with water to afford a yellow solid. Crystals of the title compound suitable for X-ray diffraction were obtained by slow evaporation of a solution of the solid in DMF at room temperature for $5 \mathrm{~d}$ (yield $1.20 \mathrm{~g}, 75 \%$; m.p: $484-485 \mathrm{~K}$ ). ${ }^{1} \mathrm{H}$ NMR (300 MHz, $\left.\mathrm{CDCl}_{3}\right): \delta 13.59(s, 2 \mathrm{H}, \mathrm{OH}), 10.14(s, 2 \mathrm{H}$, $\mathrm{OH}), 7.58-7.61(d, 2 \mathrm{H}, \mathrm{ArH}), 6.37-6.41(d, 2 \mathrm{H}, \mathrm{ArH}), 6.30$ $6.31(s, 2 \mathrm{H}, \mathrm{ArH}), 3.34\left(d, 6 \mathrm{H}, \mathrm{CH}_{3}\right)$.

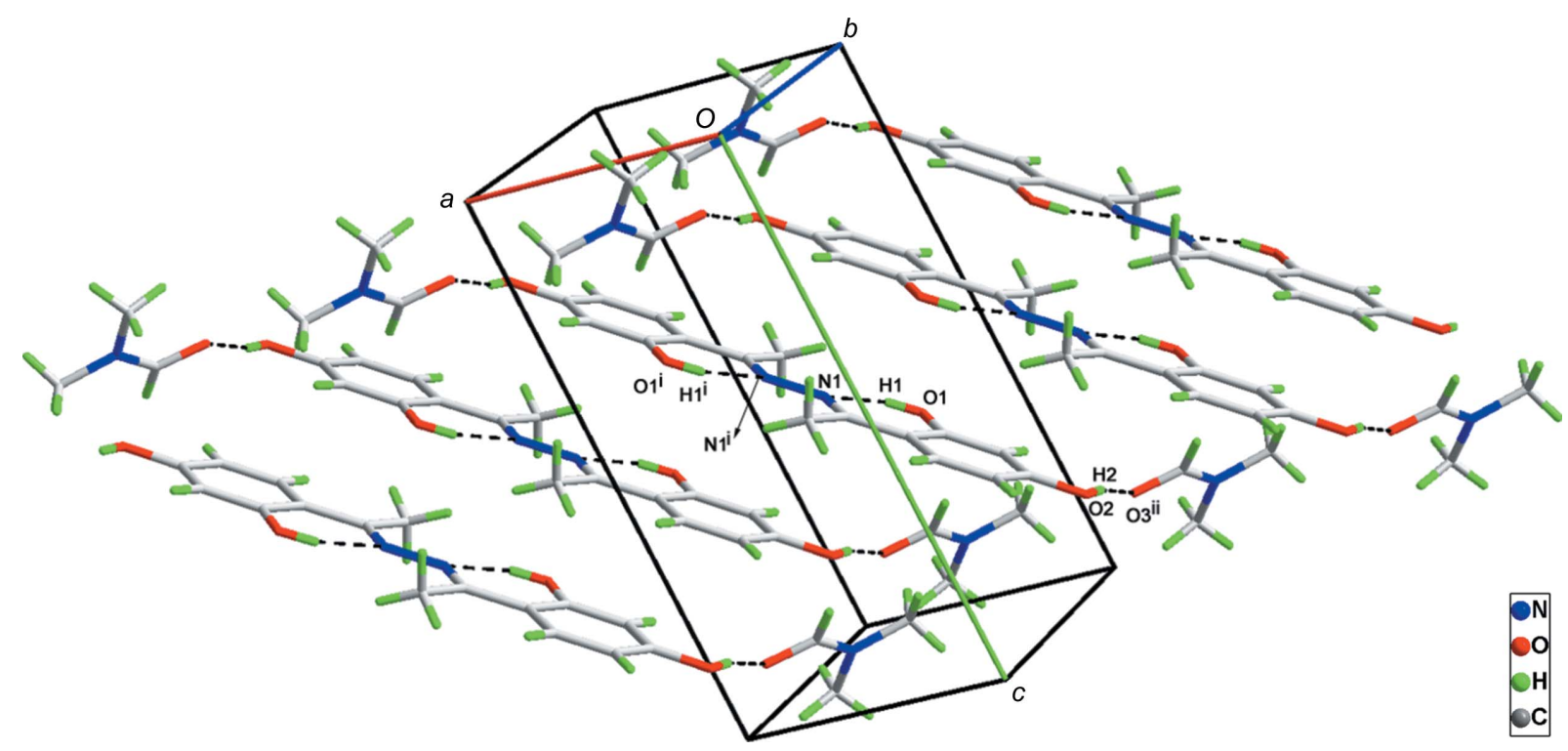

Figure 2

The crystal packing of the title compound. Hydrogen bonds are shown as dashed lines. [Symmetry codes: (i) $-x+1,-y+1,-z+1$; (ii) $x-1, y+1, z$.] 
Table 2

Experimental details.

\begin{tabular}{|c|c|}
\hline \multicolumn{2}{|l|}{ Crystal data } \\
\hline Chemical formula & $\mathrm{C}_{16} \mathrm{H}_{16} \mathrm{~N}_{2} \mathrm{O}_{4} \cdot 2 \mathrm{C}_{3} \mathrm{H}_{7} \mathrm{NO}$ \\
\hline$M_{\mathrm{r}}$ & 446.50 \\
\hline Crystal system, space group & Triclinic, $P \overline{1}$ \\
\hline Temperature (K) & 298 \\
\hline$a, b, c(\AA)$ & $6.1616(7), 7.3109(8), 13.4537(15)$ \\
\hline$\alpha, \beta, \gamma\left({ }^{\circ}\right)$ & $96.771(1), 103.049(2), 96.607(1)$ \\
\hline$V\left(\AA^{3}\right)$ & $579.96(11)$ \\
\hline$Z$ & 1 \\
\hline Radiation type & Mo $K \alpha$ \\
\hline$\mu\left(\mathrm{mm}^{-1}\right)$ & 0.09 \\
\hline Crystal size $(\mathrm{mm})$ & $0.48 \times 0.43 \times 0.21$ \\
\hline \multicolumn{2}{|l|}{ Data collection } \\
\hline Diffractometer & $\begin{array}{l}\text { Bruker SMART CCD area- } \\
\text { detector }\end{array}$ \\
\hline Absorption correction & $\begin{array}{l}\text { Multi-scan (SADABS; Sheldrick, } \\
\text { 1996) }\end{array}$ \\
\hline$T_{\min }, T_{\max }$ & $0.956,0.981$ \\
\hline $\begin{array}{l}\text { No. of measured, independent and } \\
\text { observed }[I>2 \sigma(I)] \text { reflections }\end{array}$ & $2902,2001,1313$ \\
\hline$R_{\text {int }}$ & 0.026 \\
\hline$(\sin \theta / \lambda)_{\max }\left(\AA^{-1}\right)$ & 0.595 \\
\hline \multicolumn{2}{|l|}{ Refinement } \\
\hline$R\left[F^{2}>2 \sigma\left(F^{2}\right)\right], w R\left(F^{2}\right), S$ & $0.055,0.187,1.02$ \\
\hline No. of reflections & 2001 \\
\hline No. of parameters & 149 \\
\hline H-atom treatment & H-atom parameters constrained \\
\hline$\Delta \rho_{\max }, \Delta \rho_{\min }\left(\mathrm{e} \AA^{-3}\right)$ & $0.28,-0.25$ \\
\hline
\end{tabular}

Computer programs: SMART and SAINT (Bruker, 2007), SHELXS97, SHELXL97 and SHELXTL (Sheldrick, 2008).

\section{Refinement}

Crystal data, data collection and structure refinement details are summarized in Table 2 . $\mathrm{H}$ atoms were placed geometrically $(\mathrm{C}-\mathrm{H}=0.93-0.96 \AA$ and $\mathrm{O}-\mathrm{H}=0.82 \AA)$ and refined as riding, with $U_{\text {iso }}(\mathrm{H})=1.2 U_{\text {eq }}(\mathrm{C})$ for aromatic $\mathrm{H}$ atoms or $1.5 U_{\text {eq }}(\mathrm{C}, \mathrm{O})$ for methyl and hydroxy groups.

\section{Acknowledgements}

Financial support from the Natural Science Foundation of Shanxi Province (No. 2013011011-4) is gratefully acknowledged.

\section{References}

Bruker (2007). SMART and SAINT. Bruker AXS Inc., Madison, Wisconsin, USA.

Chang, J.-G., He, G.-F. \& Li, Y.-F. (2007). Acta Cryst. E63, 03982.

Chantrapromma, S., Jansrisewangwong, P., Chanawanno, K. \& Fun, H.-K. (2011). Acta Cryst. E67, o2221-o2222.

El-Tabl, A. S., El-Saied, F. A., Plass, W. \& Al-Hakimi, A. N. (2008). Spectrochim. Acta A Mol. Biomol. Spectrosc. 71, 90-99.

Fayos, J., Martínez-Ripoll, M., García-Mina, M. C., GonzalezMartínez, J. \& Arrese, F. (1980). Acta Cryst. B36, 1952-1953.

Groom, C. R. \& Allen, F. H. (2014). Angew. Chem. Int. Ed. 53, 662671.

Guo, Y.-N., Xu, G.-F., Gamez, P., Zhao, L., Lin, S.-Y., Deng, R., Tang, J.-K. \& Zhang, H.-J. (2010). J. Am. Chem. Soc. 132, 8538-8539.

Kitaev, Y. P., Buzykin, B. I. \& Troepol'skaya, T. V. (1970). Russ. Chem. Rev. 39, 441-456.

Qin, D.-D., Yang, Z.-Y. \& Qi, G.-F. (2009). Spectrochim. Acta A Mol. Biomol. Spectrosc. 74, 415-420.

Ramamohan, L., Shikkargol, R. K., Angadi, S. D. \& Kulkarni, V. H. (1995). Asian J. Pure Appl. Chem. 1, 86-89.

Rollas, S. \& Küçükgüzel, Ş. G. (2007). Molecules, 12, 1910-1939.

Sheldrick, G. M. (1996). SADABS. University of Göttingen, Germany.

Sheldrick, G. M. (2008). Acta Cryst. A64, 112-122.

Tai, X.-S., Xu, J., Feng, Y.-M. \& Liang, Z.-P. (2008). Acta Cryst. E64, 0905.

Zhang, J.-H., Dong, W.-L., Ge, Y.-Q. \& Zhao, B.-X. (2008). Acta Cryst. E64, o166. 


\section{supporting information}

Acta Cryst. (2016). E72, 467-469 [https://doi.org/10.1107/S2056989016003686]

Crystal structure of $(E, E)-2^{\prime}, 4^{\prime}$-dihydroxyacetophenone azine dimethylformamide disolvate

Wen-Juan Li and Hong-Fei Han

Computing details

Data collection: SMART (Bruker, 2007); cell refinement: SAINT (Bruker, 2007); data reduction: SAINT (Bruker, 2007); program(s) used to solve structure: SHELXS97 (Sheldrick, 2008); program(s) used to refine structure: SHELXL97

(Sheldrick, 2008); molecular graphics: SHELXTL (Sheldrick, 2008); software used to prepare material for publication:

SHELXTL (Sheldrick, 2008).

4,4'-[1,1'-(Hydrazinediylidene)bis(ethan-1-yl-1-ylidene)]bis(benzene-1,3-diol)

Crystal data

$\mathrm{C}_{16} \mathrm{H}_{16} \mathrm{~N}_{2} \mathrm{O}_{4} \cdot 2 \mathrm{C}_{3} \mathrm{H}_{7} \mathrm{NO}$

$M_{r}=446.50$

Triclinic, $P \overline{1}$

Hall symbol: -P 1

$a=6.1616(7) \AA$

$b=7.3109(8) \AA$

$c=13.4537(15) \AA$

$\alpha=96.771(1)^{\circ}$

$\beta=103.049(2)^{\circ}$

$\gamma=96.607(1)^{\circ}$

$V=579.96(11) \AA^{3}$

Data collection

Bruker SMART CCD area-detector diffractometer

Radiation source: fine-focus sealed tube Graphite monochromator

phi and $\omega$ scans

Absorption correction: multi-scan

(SADABS; Sheldrick, 1996)

$T_{\min }=0.956, T_{\max }=0.981$

Refinement

Refinement on $F^{2}$

Least-squares matrix: full

$R\left[F^{2}>2 \sigma\left(F^{2}\right)\right]=0.055$

$w R\left(F^{2}\right)=0.187$

$S=1.02$

2001 reflections

149 parameters

0 restraints
$Z=1$

$F(000)=238$

$D_{\mathrm{x}}=1.278 \mathrm{Mg} \mathrm{m}^{-3}$

Mo $K \alpha$ radiation, $\lambda=0.71073 \AA$

Cell parameters from 1119 reflections

$\theta=3.0-26.5^{\circ}$

$\mu=0.09 \mathrm{~mm}^{-1}$

$T=298 \mathrm{~K}$

Block, colorless

$0.48 \times 0.43 \times 0.21 \mathrm{~mm}$

2902 measured reflections

2001 independent reflections

1313 reflections with $I>2 \sigma(I)$

$R_{\text {int }}=0.026$

$\theta_{\max }=25.0^{\circ}, \theta_{\min }=3.0^{\circ}$

$h=-6 \rightarrow 7$

$k=-8 \rightarrow 7$

$l=-15 \rightarrow 15$

Primary atom site location: structure-invariant direct methods

Secondary atom site location: difference Fourier map

Hydrogen site location: inferred from neighbouring sites

$\mathrm{H}$-atom parameters constrained 
$w=1 /\left[\sigma^{2}\left(F_{\mathrm{o}}^{2}\right)+(0.1079 P)^{2}+0.0859 P\right]$

where $P=\left(F_{\mathrm{o}}^{2}+2 F_{\mathrm{c}}^{2}\right) / 3$

$(\Delta / \sigma)_{\max }<0.001$

$\Delta \rho_{\max }=0.28$ e $\AA^{-3}$
$\Delta \rho_{\min }=-0.25$ e $\AA^{-3}$

Extinction correction: SHELXL97 (Sheldrick, 2008), $\mathrm{Fc}^{*}=\mathrm{kFc}\left[1+0.001 \mathrm{xFc}^{2} \lambda^{3} / \sin (2 \theta)\right]^{-1 / 4}$

Extinction coefficient: $0.17(2)$

\section{Special details}

Geometry. All esds (except the esd in the dihedral angle between two 1.s. planes) are estimated using the full covariance matrix. The cell esds are taken into account individually in the estimation of esds in distances, angles and torsion angles; correlations between esds in cell parameters are only used when they are defined by crystal symmetry. An approximate (isotropic) treatment of cell esds is used for estimating esds involving l.s. planes.

Refinement. Refinement of $\mathrm{F}^{2}$ against ALL reflections. The weighted R-factor $\mathrm{wR}$ and goodness of fit $\mathrm{S}$ are based on $\mathrm{F}^{2}$, conventional R-factors $R$ are based on $F$, with $F$ set to zero for negative $F^{2}$. The threshold expression of $F^{2}>2 \operatorname{sigma}\left(F^{2}\right)$ is used only for calculating R-factors(gt) etc. and is not relevant to the choice of reflections for refinement. R-factors based on $\mathrm{F}^{2}$ are statistically about twice as large as those based on F, and R- factors based on ALL data will be even larger.

Fractional atomic coordinates and isotropic or equivalent isotropic displacement parameters $\left(\AA^{2}\right)$

\begin{tabular}{|c|c|c|c|c|}
\hline & $x$ & $y$ & $z$ & $U_{\text {iso }} * / U_{\text {eq }}$ \\
\hline N1 & $0.4279(3)$ & $0.5237(2)$ & $0.53085(13)$ & $0.0391(5)$ \\
\hline $\mathrm{N} 2$ & $0.5727(4)$ & $0.1587(3)$ & $0.91556(16)$ & $0.0566(6)$ \\
\hline $\mathrm{O} 1$ & $0.2575(3)$ & $0.7659(2)$ & $0.62846(12)$ & $0.0537(5)$ \\
\hline H1 & 0.3372 & 0.7270 & 0.5919 & $0.081^{*}$ \\
\hline $\mathrm{O} 2$ & $-0.2843(3)$ & $0.5582(3)$ & $0.80524(14)$ & $0.0679(6)$ \\
\hline $\mathrm{H} 2$ & -0.2793 & 0.6712 & 0.8190 & $0.102^{*}$ \\
\hline $\mathrm{O} 3$ & $0.7469(4)$ & $-0.0813(3)$ & $0.87057(18)$ & $0.0899(8)$ \\
\hline $\mathrm{C} 1$ & $0.3219(5)$ & $0.1857(3)$ & $0.5203(2)$ & $0.0574(7)$ \\
\hline $\mathrm{H} 1 \mathrm{~A}$ & 0.2229 & 0.1462 & 0.4531 & $0.086^{*}$ \\
\hline H1B & 0.2779 & 0.1098 & 0.5681 & $0.086^{*}$ \\
\hline $\mathrm{H} 1 \mathrm{C}$ & 0.4737 & 0.1732 & 0.5172 & $0.086^{*}$ \\
\hline $\mathrm{C} 2$ & $0.3080(3)$ & $0.3858(3)$ & $0.55569(15)$ & $0.0374(6)$ \\
\hline $\mathrm{C} 3$ & $0.1542(3)$ & $0.4334(3)$ & $0.62014(15)$ & $0.0368(6)$ \\
\hline $\mathrm{C} 4$ & $0.1368(4)$ & $0.6208(3)$ & $0.65420(16)$ & $0.0403(6)$ \\
\hline $\mathrm{C} 5$ & $-0.0090(4)$ & $0.6624(3)$ & $0.71594(16)$ & $0.0458(6)$ \\
\hline H5 & -0.0180 & 0.7861 & 0.7380 & $0.055^{*}$ \\
\hline C6 & $-0.1404(4)$ & $0.5221(4)$ & $0.74482(17)$ & $0.0479(6)$ \\
\hline $\mathrm{C} 7$ & $-0.1270(4)$ & $0.3382(4)$ & $0.71238(18)$ & $0.0528(7)$ \\
\hline $\mathrm{H} 7$ & -0.2153 & 0.2431 & 0.7316 & $0.063^{*}$ \\
\hline $\mathrm{C} 8$ & $0.0176(4)$ & $0.2968(3)$ & $0.65154(17)$ & $0.0472(6)$ \\
\hline H8 & 0.0249 & 0.1723 & 0.6304 & $0.057^{*}$ \\
\hline C9 & $0.5795(5)$ & $-0.0016(5)$ & $0.8617(2)$ & $0.0696(9)$ \\
\hline H9 & 0.4489 & -0.0589 & 0.8136 & $0.084 *$ \\
\hline $\mathrm{C} 10$ & $0.7669(5)$ & $0.2551(5)$ & $0.9909(2)$ & $0.0808(9)$ \\
\hline $\mathrm{H} 10 \mathrm{~A}$ & 0.8729 & 0.1706 & 1.0084 & $0.121 *$ \\
\hline $\mathrm{H} 10 \mathrm{~B}$ & 0.7228 & 0.3039 & 1.0516 & $0.121^{*}$ \\
\hline $\mathrm{H} 10 \mathrm{C}$ & 0.8355 & 0.3556 & 0.9632 & $0.121^{*}$ \\
\hline C11 & $0.3699(5)$ & $0.2460(5)$ & 0.8988 & $0.0889(10)$ \\
\hline H11A & 0.2480 & 0.1611 & 0.8530 & $0.133^{*}$ \\
\hline H11B & 0.3951 & 0.3568 & 0.8687 & $0.133^{*}$ \\
\hline $\mathrm{H} 11 \mathrm{C}$ & 0.3324 & 0.2778 & 0.9635 & $0.133^{*}$ \\
\hline
\end{tabular}


Atomic displacement parameters $\left(\AA^{2}\right)$

\begin{tabular}{lllllll}
\hline & $U^{11}$ & $U^{22}$ & $U^{33}$ & $U^{12}$ & $U^{13}$ & $U^{23}$ \\
\hline $\mathrm{N} 1$ & $0.0427(11)$ & $0.0333(11)$ & $0.0416(10)$ & $0.0063(8)$ & $0.0117(8)$ & $0.0035(8)$ \\
$\mathrm{N} 2$ & $0.0545(13)$ & $0.0620(15)$ & $0.0553(12)$ & $0.0128(11)$ & $0.0150(10)$ & $0.0088(11)$ \\
O1 & $0.0704(11)$ & $0.0331(10)$ & $0.0647(11)$ & $0.0015(8)$ & $0.0367(9)$ & $0.0024(7)$ \\
O2 & $0.0738(13)$ & $0.0718(14)$ & $0.0713(12)$ & $0.0124(10)$ & $0.0428(10)$ & $0.0122(10)$ \\
O3 & $0.0897(16)$ & $0.0827(17)$ & $0.1017(17)$ & $0.0266(14)$ & $0.0354(13)$ & $-0.0059(13)$ \\
C1 & $0.0708(17)$ & $0.0345(14)$ & $0.0745(17)$ & $0.0076(12)$ & $0.0332(14)$ & $0.0080(12)$ \\
C2 & $0.0380(12)$ & $0.0337(12)$ & $0.0377(11)$ & $0.0037(9)$ & $0.0037(9)$ & $0.0065(9)$ \\
C3 & $0.0383(12)$ & $0.0346(12)$ & $0.0351(11)$ & $0.0028(9)$ & $0.0048(9)$ & $0.0057(9)$ \\
C4 & $0.0450(13)$ & $0.0378(13)$ & $0.0364(11)$ & $0.0024(10)$ & $0.0078(10)$ & $0.0064(9)$ \\
C5 & $0.0532(14)$ & $0.0414(14)$ & $0.0427(12)$ & $0.0066(11)$ & $0.0136(11)$ & $0.0020(10)$ \\
C6 & $0.0451(13)$ & $0.0583(16)$ & $0.0411(12)$ & $0.0065(11)$ & $0.0120(10)$ & $0.0081(11)$ \\
C7 & $0.0548(15)$ & $0.0502(16)$ & $0.0561(15)$ & $-0.0022(12)$ & $0.0199(12)$ & $0.0155(11)$ \\
C8 & $0.0539(14)$ & $0.0385(14)$ & $0.0497(13)$ & $0.0031(11)$ & $0.0137(11)$ & $0.0102(10)$ \\
C9 & $0.0675(19)$ & $0.081(2)$ & $0.0575(16)$ & $-0.0021(16)$ & $0.0183(14)$ & $0.0061(15)$ \\
C10 & $0.078(2)$ & $0.075(2)$ & $0.078(2)$ & $0.0069(17)$ & $0.0043(17)$ & $0.0000(16)$ \\
C11 & $0.069(2)$ & $0.102(3)$ & $0.102(2)$ & $0.0268(19)$ & $0.0191(18)$ & $0.032(2)$ \\
& & & & &
\end{tabular}

Geometric parameters $\left(\AA,{ }^{\circ}\right)$

\begin{tabular}{|c|c|c|c|}
\hline $\mathrm{N} 1-\mathrm{C} 2$ & $1.301(3)$ & $\mathrm{C} 3-\mathrm{C} 4$ & $1.417(3)$ \\
\hline $\mathrm{N} 1-\mathrm{N} 1^{\mathrm{i}}$ & $1.391(3)$ & $\mathrm{C} 4-\mathrm{C} 5$ & $1.389(3)$ \\
\hline $\mathrm{N} 2-\mathrm{C} 9$ & $1.313(4)$ & $\mathrm{C} 5-\mathrm{C} 6$ & $1.380(3)$ \\
\hline $\mathrm{N} 2-\mathrm{C} 10$ & $1.430(4)$ & $\mathrm{C} 5-\mathrm{H} 5$ & 0.9300 \\
\hline $\mathrm{N} 2-\mathrm{C} 11$ & $1.453(3)$ & $\mathrm{C} 6-\mathrm{C} 7$ & $1.381(3)$ \\
\hline $\mathrm{O} 1-\mathrm{C} 4$ & $1.349(3)$ & $\mathrm{C} 7-\mathrm{C} 8$ & $1.374(3)$ \\
\hline $\mathrm{O} 1-\mathrm{H} 1$ & 0.8200 & $\mathrm{C} 7-\mathrm{H} 7$ & 0.9300 \\
\hline $\mathrm{O} 2-\mathrm{C} 6$ & $1.358(3)$ & $\mathrm{C} 8-\mathrm{H} 8$ & 0.9300 \\
\hline $\mathrm{O} 2-\mathrm{H} 2$ & 0.8200 & C9- $\mathrm{H} 9$ & 0.9300 \\
\hline $\mathrm{O} 3-\mathrm{C} 9$ & $1.232(3)$ & $\mathrm{C} 10-\mathrm{H} 10 \mathrm{~A}$ & 0.9600 \\
\hline $\mathrm{C} 1-\mathrm{C} 2$ & $1.503(3)$ & $\mathrm{C} 10-\mathrm{H} 10 \mathrm{~B}$ & 0.9600 \\
\hline $\mathrm{C} 1-\mathrm{H} 1 \mathrm{~A}$ & 0.9600 & $\mathrm{C} 10-\mathrm{H} 10 \mathrm{C}$ & 0.9600 \\
\hline $\mathrm{C} 1-\mathrm{H} 1 \mathrm{~B}$ & 0.9600 & $\mathrm{C} 11-\mathrm{H} 11 \mathrm{~A}$ & 0.9600 \\
\hline $\mathrm{C} 1-\mathrm{H} 1 \mathrm{C}$ & 0.9600 & $\mathrm{C} 11-\mathrm{H} 11 \mathrm{~B}$ & 0.9600 \\
\hline $\mathrm{C} 2-\mathrm{C} 3$ & $1.465(3)$ & $\mathrm{C} 11-\mathrm{H} 11 \mathrm{C}$ & 0.9600 \\
\hline $\mathrm{C} 3-\mathrm{C} 8$ & $1.396(3)$ & & \\
\hline $\mathrm{C} 2-\mathrm{N} 1-\mathrm{N} 1^{\mathrm{i}}$ & $116.3(2)$ & $\mathrm{O} 2-\mathrm{C} 6-\mathrm{C} 5$ & $122.1(2)$ \\
\hline $\mathrm{C} 9-\mathrm{N} 2-\mathrm{C} 10$ & $120.9(2)$ & $\mathrm{O} 2-\mathrm{C} 6-\mathrm{C} 7$ & $118.0(2)$ \\
\hline $\mathrm{C} 9-\mathrm{N} 2-\mathrm{C} 11$ & $121.2(3)$ & $\mathrm{C} 5-\mathrm{C} 6-\mathrm{C} 7$ & $119.9(2)$ \\
\hline $\mathrm{C} 10-\mathrm{N} 2-\mathrm{C} 11$ & $117.9(3)$ & $\mathrm{C} 8-\mathrm{C} 7-\mathrm{C} 6$ & $119.6(2)$ \\
\hline $\mathrm{C} 4-\mathrm{O} 1-\mathrm{H} 1$ & 109.5 & $\mathrm{C} 8-\mathrm{C} 7-\mathrm{H} 7$ & 120.2 \\
\hline $\mathrm{C} 6-\mathrm{O} 2-\mathrm{H} 2$ & 109.5 & $\mathrm{C} 6-\mathrm{C} 7-\mathrm{H} 7$ & 120.2 \\
\hline $\mathrm{C} 2-\mathrm{C} 1-\mathrm{H} 1 \mathrm{~A}$ & 109.5 & $\mathrm{C} 7-\mathrm{C} 8-\mathrm{C} 3$ & $122.9(2)$ \\
\hline $\mathrm{C} 2-\mathrm{C} 1-\mathrm{H} 1 \mathrm{~B}$ & 109.5 & $\mathrm{C} 7-\mathrm{C} 8-\mathrm{H} 8$ & 118.6 \\
\hline $\mathrm{H} 1 \mathrm{~A}-\mathrm{C} 1-\mathrm{H} 1 \mathrm{~B}$ & 109.5 & $\mathrm{C} 3-\mathrm{C} 8-\mathrm{H} 8$ & 118.6 \\
\hline
\end{tabular}




$\begin{array}{llll}\mathrm{C} 2-\mathrm{C} 1-\mathrm{H} 1 \mathrm{C} & 109.5 & \mathrm{O} 3-\mathrm{C} 9-\mathrm{N} 2 & 124.5(3) \\ \mathrm{H} 1 \mathrm{~A}-\mathrm{C} 1-\mathrm{H} 1 \mathrm{C} & 109.5 & \mathrm{O} 3-\mathrm{C} 9-\mathrm{H} 9 & 117.8 \\ \mathrm{H} 1 \mathrm{~B}-\mathrm{C} 1-\mathrm{H} 1 \mathrm{C} & 109.5 & \mathrm{~N} 2-\mathrm{C} 9-\mathrm{H} 9 & 117.8 \\ \mathrm{~N} 1-\mathrm{C} 2-\mathrm{C} 3 & 116.96(19) & \mathrm{N} 2-\mathrm{C} 10-\mathrm{H} 10 \mathrm{~A} & 109.5 \\ \mathrm{~N} 1-\mathrm{C} 2-\mathrm{C} 1 & 122.62(19) & \mathrm{N} 2-\mathrm{C} 10-\mathrm{H} 10 \mathrm{~B} & 109.5 \\ \mathrm{C} 3-\mathrm{C} 2-\mathrm{C} 1 & 120.4(2) & \mathrm{H} 10 \mathrm{~A}-\mathrm{C} 10-\mathrm{H} 10 \mathrm{~B} & 109.5 \\ \mathrm{C} 8-\mathrm{C} 3-\mathrm{C} 4 & 116.5(2) & \mathrm{N} 2-\mathrm{C} 10-\mathrm{H} 10 \mathrm{C} & 109.5 \\ \mathrm{C} 8-\mathrm{C} 3-\mathrm{C} 2 & 121.9(2) & \mathrm{H} 10 \mathrm{~A}-\mathrm{C} 10-\mathrm{H} 10 \mathrm{C} & 109.5 \\ \mathrm{C} 4-\mathrm{C} 3-\mathrm{C} 2 & 121.6(2) & \mathrm{H} 10 \mathrm{~B}-\mathrm{C} 10-\mathrm{H} 10 \mathrm{C} & 109.5 \\ \mathrm{O} 1-\mathrm{C} 4-\mathrm{C} 5 & 117.0(2) & \mathrm{N} 2-\mathrm{C} 11-\mathrm{H} 11 \mathrm{~A} & 109.5 \\ \mathrm{O} 1-\mathrm{C} 4-\mathrm{C} 3 & 122.42(19) & \mathrm{N} 2-\mathrm{C} 11-\mathrm{H} 11 \mathrm{~B} & 109.5 \\ \mathrm{C} 5-\mathrm{C} 4-\mathrm{C} 3 & 120.6(2) & \mathrm{H} 11 \mathrm{~A}-\mathrm{C} 11-\mathrm{H} 11 \mathrm{~B} & 109.5 \\ \mathrm{C} 6-\mathrm{C} 5-\mathrm{C} 4 & 120.7(2) & \mathrm{N} 2-\mathrm{C} 11-\mathrm{H} 11 \mathrm{C} & 109.5 \\ \mathrm{C} 6-\mathrm{C} 5-\mathrm{H} 5 & 119.7 & \mathrm{H} 11 \mathrm{~A}-\mathrm{C} 11-\mathrm{H} 11 \mathrm{C} & 109.5 \\ \mathrm{C} 4-\mathrm{C} 5-\mathrm{H} 5 & 119.7 & \mathrm{H} 11 \mathrm{~B}-\mathrm{C} 11-\mathrm{H} 11 \mathrm{C} & 109.5 \\ & & & \\ \mathrm{~N} 1-\mathrm{N} 1-\mathrm{C} 2-\mathrm{C} 3 & -179.63(19) & \mathrm{C} 3-\mathrm{C} 4-\mathrm{C} 5-\mathrm{C} 6 & -0.3(3) \\ \mathrm{N} 1-\mathrm{N} 1-\mathrm{C} 2-\mathrm{C} 1 & -0.1(3) & \mathrm{C} 4-\mathrm{C} 5-\mathrm{C} 6-\mathrm{O} 2 & 179.9(2) \\ \mathrm{N} 1-\mathrm{C} 2-\mathrm{C} 3-\mathrm{C} 8 & -179.94(18) & \mathrm{C} 4-\mathrm{C} 5-\mathrm{C} 6-\mathrm{C} 7 & 0.2(3) \\ \mathrm{C} 1-\mathrm{C} 2-\mathrm{C} 3-\mathrm{C} 8 & 0.5(3) & \mathrm{O} 2-\mathrm{C} 6-\mathrm{C} 7-\mathrm{C} 8 & -179.7(2) \\ \mathrm{N} 1-\mathrm{C} 2-\mathrm{C} 3-\mathrm{C} 4 & -0.2(3) & \mathrm{C} 5-\mathrm{C} 6-\mathrm{C} 7-\mathrm{C} 8 & 0.0(4) \\ \mathrm{C} 1-\mathrm{C} 2-\mathrm{C} 3-\mathrm{C} 4 & -179.69(19) & \mathrm{C} 6-\mathrm{C} 7-\mathrm{C} 8-\mathrm{C} 3 & -0.1(4) \\ \mathrm{C} 8-\mathrm{C} 3-\mathrm{C} 4-\mathrm{O} 1 & -179.28(19) & \mathrm{C} 4-\mathrm{C} 3-\mathrm{C} 8-\mathrm{C} 7 & -0.1(3) \\ \mathrm{C} 2-\mathrm{C} 3-\mathrm{C} 4-\mathrm{O} 1 & 0.9(3) & \mathrm{C} 2-\mathrm{C} 3-\mathrm{C} 8-\mathrm{C} 7 & 179.7(2) \\ \mathrm{C} 8-\mathrm{C} 3-\mathrm{C} 4-\mathrm{C} 5 & 0.3(3) & \mathrm{C} 10-\mathrm{N} 2-\mathrm{C} 9-\mathrm{O} 3 & 0.1(4) \\ \mathrm{C} 2-\mathrm{C} 3-\mathrm{C} 4-\mathrm{C} 5 & -179.47(19) & \mathrm{C} 11-\mathrm{N} 2-\mathrm{C} 9-\mathrm{O} 3 & 178.5(3) \\ \mathrm{O} 1-\mathrm{C} 4-\mathrm{C} 5-\mathrm{C} 6 & 179.3(2) & & \end{array}$

Symmetry code: (i) $-x+1,-y+1,-z+1$.

Hydrogen-bond geometry $\left(\AA,{ }^{\circ}\right)$

\begin{tabular}{lllll}
\hline$D-\mathrm{H} \cdots A$ & $D-\mathrm{H}$ & $\mathrm{H} \cdots A$ & $D \cdots A$ & $D-\mathrm{H} \cdots A$ \\
\hline $\mathrm{O} 1-\mathrm{H} 1 \cdots \mathrm{N} 1$ & 0.82 & 1.82 & $2.543(2)$ & 147 \\
$\mathrm{O} 2-\mathrm{H} 2 \cdots \mathrm{O} 3^{\mathrm{ii}}$ & 0.82 & 1.84 & $2.649(3)$ & 171 \\
\hline
\end{tabular}

Symmetry code: (ii) $x-1, y+1, z$. 